\title{
Charge Migration Efficiency Optimization in Hybrid Electrical Energy Storage (HEES) Systems
}

\begin{abstract}
Electrical energy is high-quality form of energy, and thus it is beneficial to store the excessive electric energy in the electrical energy storage (EES) rather than converting into a different type of energy. Like memory devices, no single type of EES element can fulfill all the desirable requirements. Despite active research on the new EES technologies, it is not likely to have an ultimate high-efficiency, highpower/energy capacity, low-cost, and long-cycle life EES element in the near future. We propose an HEES system that consists of two or more heterogeneous EES elements, thereby realizing the advantages of each EES element while hiding their weaknesses. The HEES management problems can be broken into charge allocation into different banks of EES elements, charge replacement (i.e., discharge) from different banks of EES elements, and charge migration from one bank to another bank of EES elements.

In spite of the optimal charge allocation and replacement, charge migration is mandatory to leverage the EES system efficiency. This paper is the first paper that formally describes the charge migration efficiency and its optimization. We first define the charge migration architecture and the corresponding charge migration problem. We provide a systematic solution for a single source and single destination charge migration considering the efficiency of the charger and power converter, the rate capacity effect of the storage element, the terminal voltage variation of the storage element as a function of the state of charge (SoC), and so on. Experimental results for an HEES system comprising of banks of batteries and supercapacitors demonstrate a migration efficiency improvement up to $51.3 \%$, for supercapacitor to battery and supercapacitor to supercapacitor charge migration.
\end{abstract}

\section{INTRODUCTION}

Electrical energy usage changes over time due to the types of load devices and user behaviors. Fossil fuel power plants and nuclear power plants can generate steady amount of power and also control the amount of power generation. On the other hand, the output power levels of most renewable power sources are not controllable and are largely dependent on the environmental factors (e.g., the irradiance level or the climate condition.) Even for the fossil fuel and nuclear power plants, the amount of generation cannot be changed quickly enough to respond to rapid changes in the load demand. Therefore, electricity supply (generation) and demand (consumption) are typically not balanced with each other. Storage of excessive energy and compensation of the energy shortage (or avoiding waste of energy) can significantly mitigate the under (or over)-investment in the generation facilities. Electrical energy storage (EES) systems can thus increase power reliability and efficiency, compensate the supply-demand mismatch, and regulate the peak-power demand.

Permission to make digital or hard copies of all or part of this work for personal or classroom use is granted without fee provided that copies are not made or distributed for profit or commercial advantage and that copies bear this notice and the full citation on the first page. To copy otherwise, to republish, to post on servers or to redistribute to lists, requires prior specific permission and/or a fee.

ISLPED'11, August 1-3, 2011, Fukuoka, Japan.

Copyright 2011 ACM X-XXXXX-XX-X/XX/XX ...\$10.00.
Electrical energy is a high-quality form of energy [1] in the sense that they can be easily and efficiently converted into other forms of energy and furthermore it can be used to control other forms of energy. More precisely, the energy quality factor may be used to describe how much energy is consumed to produce another form of energy. Electrical energy can be efficiently transformed into other forms of energy, with a quality factor of $1.59 \times 10^{5} \mathrm{seJ} / \mathrm{J}$ (solar emjoules per joule) which is 2-4 times higher than that of fossil fuel energy [2]. Although storage cost per unit energy for electrical energy is higher than that of thermal or kinetic energy [3], storage of electrical energy in the form of electrical (or electrochemical) energy has advantages in terms of higher cycle efficiency (i.e., efficiency during a deposit and retrieval cycle) and faster response time (i.e., time it takes to ramp up or down the power level.)

There are examples of actual deployment of a grid-scale EES system to mitigate the gap between the supply and demand $[4,5]$. In addition, most stand-alone renewable energy sources, such as solar energy, wind power, and hydropower, require an EES system. Most importantly, however, current EES systems are mainly homogeneous [5], that is, they consist of a single type of EES elements, and therefore, tend to suffer from the limitations and shortcomings of that EES element. One way to improve the performance of such EES systems is to exploit different types of EES elements with their unique strengths and weaknesses, and come up with the hybrid EES system architecture and control policies that improve the key performance characteristics of the storage system.

Let us recall the concept of memory hierarchy; no single type of memory device can fulfill all the desirable requirements such as speed, capacity, cost, non-volatility, power consumption, etc. A memory system hierarchy consists of heterogeneous types of memory devices in order to hide drawbacks of each memory type while utilizing their benefits. Although intensive research in academia and industry has focused on improving the memory technologies, it is unlikely that a single type of memory device will dominate the whole memory systems in the near future. In parallel, a lot of research and development effort has focused on the design of more efficient memory hierarchies to meet the specific needs of different computing systems and applications running on them.

Like memory devices, no single EES element can fulfill all the requirements of electrical energy storage and retrieval operations. In fact, it is not likely to have an ultimate high-efficiency, highpower/energy capacity, low-cost, and long-cycle life EES element any time soon. An HEES (hybrid EES) system is an EES system that consists of two or more heterogeneous EES elements. A simple structure of HEES systems is found in advanced electric vehicles, especially for efficient regenerative braking systems. More recently, generalized HEES systems are introduced [6, 7].

Analogous to memory management in computer systems, the energy management issues in HEES systems can be broken into charge allocation, charge replacement, and charge migration operations [6]. Power sources (and load devices) have different characteristics in terms of energy/power capacity (and voltage/current requirements.) Moreover, the cycle efficiency and output power availability of each EES element may vary depending on a number of factors, including the SoC of the element, the rate at which energy is provided or extracted, and so on [6]. Therefore, it is crucial to provide policies for charge allocation to the best-suited EES element for a given incoming power source, and for charge replacement from the best-suited 


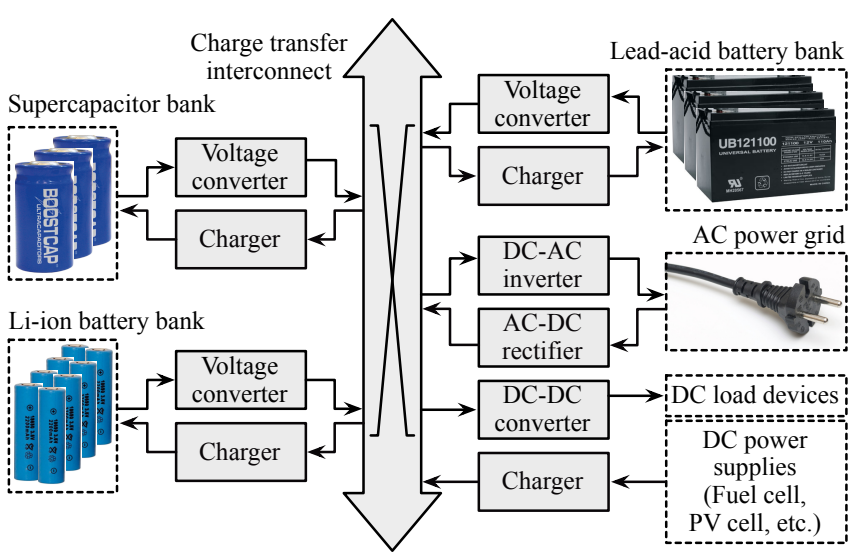

Figure 1: Architecture of the proposed HEES system.

EES element for the load device.

Even if the optimal charge allocation and replacement policies are put in place and executed, charge migration that moves charge from one EES element to another is mandatory to improve the EES system efficiency and responsiveness. Charge migration can ensure the availability of the best-suited EES element to service a load demand (as much as it is possible to do so.) This means that the chosen EES element will have the desired characteristics (in terms of its selfleakage, output power rating) with respect to the load demand.

This paper is the first paper that formally describes the charge migration efficiency and its optimization. We first define the charge migration architecture and the corresponding charge migration problem. We provide a systematic solution for a single source and a single destination charge migration considering the efficiency of the charger and power converter, rate capacity effect of the storage element, terminal voltage variation of the storage element by the state of charge (SoC), and so on. We demonstrate the optimal charge migration policy and method on two representative test cases: supercapacitor to battery, supercapcitor to supercapacitor charge migration. The experimental results show that optimization achieves a significant energy saving up to $51.3 \%$ compared with baseline charge migration methods.

\section{HYBRID ELECTRICAL ENERGY STOR- AGE SYSTEMS}

\subsection{Hybrid Electrical Energy Storage Systems}

An illustration of the proposed HEES architecture is shown in Figure 1 . The system comprises of multiple storage banks, connected to each other through local power converters (i.e., distributed voltage conversion and current regulation circuitry) and a migration interconnect. A storage bank itself is composed of homogeneous EES elements. Because the state-of-charge (SoC), terminal output voltage, and power rating of different EES banks may not be compatible with each other, direct connection among EES banks is generally not feasible. Note that even if the EES banks have the same nominal terminal voltage in the case of batteries, their actual output voltage may vary significantly depending on their SoC. The terminal voltage of a supercapacitor bank is proportional to its SoC. The migration interconnect is provided for energy transfer from the power supply, to the load device, and between the storage banks. As a result, there is a strong need for voltage conversion to hook up a storage bank to the migration interconnect and discharge the storage bank. Charging process, i.e., the current flow from the migration interconnect to the storage bank, also mandates current regulation circuitry.

As stated earlier, each EES element in existence today has its

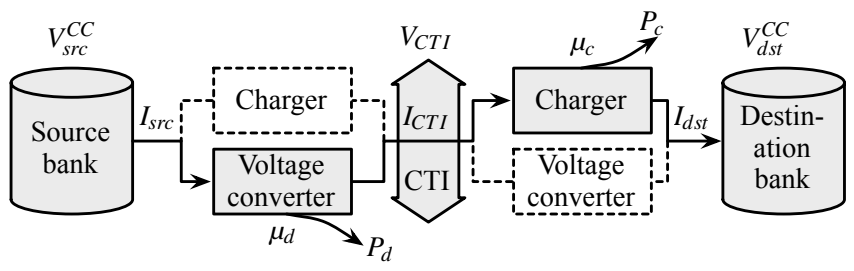

Figure 2: Single-source-single-destination charge migration.

strengths and weaknesses in such a way that there is no single EES element that fulfills all requirements of an ideal EES system, in terms of low capital cost, high cycle efficiency, long cycle life, low selfdischarge rate, and high power and energy densities. Utilization of heterogeneous EES banks within an energy storage hierarchy along with appropriate charge management circuitry and policies is one way to realize the attributes of an ideal EES system through appropriate allocation, organization, and management of different types of EES banks.

In [6], three HEES management problems are introduced: charge allocation, charge replacement, and charge migration. The charge allocation problem is, when energy comes from the external power source, to find the appropriate destination banks and energy amount that maximize the energy efficiency. Considering the characteristics and current state of the storage banks, and expected power and energy values of incoming energy, we determine the optimal charging policy. The charge replacement problem is the opposite: we determine the appropriate source banks and energy amount when there is a power demand from the load device.

\subsection{Charge Migration}

While charge allocation and replacement deal with energy exchange with external power supply and load demand, charge migration is an internal energy transfer from one EES bank to another. We see strong motivations to perform charge migration to enhance the EES system performance. First, appropriate charge migration can improve the energy efficiency by controlling the SoC of the EES banks for future charge allocation or replacement. For example, a future charge allocation may provide the best efficiency with a particular EES bank. If such EES bank has full SoC at this moment, the future charge allocation cannot transfer the charge to that EES bank unless there will be charge replacement from that bank in the mean time. Second, we should not let a leaky EES bank, e.g., a supercapacitor bank, store charge for a long period of time. We had better perform charge migration from a supercapacitor bank to another if we foresee that the charge in the supercapacitor bank will not be replaced in the near future. Third, charge migration can maximize the availability of the EES system. An EES system cannot satisfy a load demand even if the total amount of charge in the EES system is enough for the load demand because of lack of power capacity. In other words, an EES system is not available for a high-power load demand if an EES bank with a high power capacity is empty, and another EES bank with a low power capacity has full SoC. If we are able to estimate the future load demand, we may perform charge migration in advance and make the EES system available. In addition, charge migration also help enhance other EES performance metrics. In this paper, we primarily mention the charge migration problem to enhance the migration efficiency.

Figure 2 presents a basic charge migration process, from a single source bank to a single destination bank through a charge transfer interconnect (CTI). Each storage bank is connected to the CTI through a charger and a voltage converter. Although a single source and a single destination charge migration does not require the voltage converter, the voltage converter is mandatory for charge replacement as well as for keeping the voltage of the CTI controllable. Charg- 


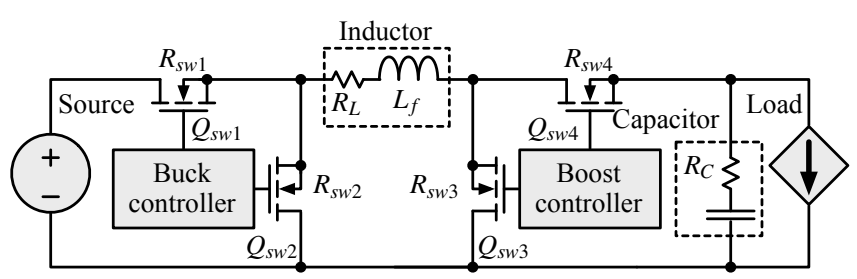

Figure 3: Buck-boost converter architecture.

ers control the amount of current through the destination EES bank. Figure 2 is a conceptual architecture to define a single source and a single destination charge migration. However, practical EES systems may have a complicated interconnect architecture, which is out of focus of this paper.

The open circuit terminal voltage (OCV) of the source EES bank and the OCV of the destination EES banks at time $t$ are denoted by $V_{s r c}^{O C}(t)$ and $V_{d s t}^{O C}(t)$, respectively. Such OCV values are functions of time $t$ since they depend on the SoCs of source and destination EES banks, which change over time $t$. Similarly, we denote the closed circuit terminal voltage (CCV) of the source and destination at time $t$ by $V_{s r c}^{C C}(t)$ and $V_{d s t}^{C C}(t)$, respectively. The OCV and CCV are generally different from each other due to the internal series resistance.

We call the CTI voltage migration voltage, which is denoted by $V_{C T I}(t)$. We denote important current flows such as the discharging current from the source to the discharger, migration current on the CTI from the discharger to the charger, and charging current from the charger to the destination at time $t$, as $I_{s r c}(t), I_{C T I}(t)$, and $I_{d s t}(t)$, respectively. Moreover, the efficiencies of the charger and discharger at time $t$ are denoted by $\eta_{c}(t)$ and $\eta_{d}(t)$, respectively, and defined by

$$
\eta_{c}(t)=\frac{V_{d s t}^{C C}(t) \cdot I_{d s t}(t)}{V_{C T I}(t) \cdot I_{C T I}(t)}, \quad \eta_{d}(t)=\frac{V_{C T I}(t) \cdot I_{C T I}(t)}{V_{s r C}^{C C}(t) \cdot I_{s r C}(t)}
$$

Finally, the overall migration efficiency $\eta_{m}(t)$ is defined by

$$
\eta_{m}(t)=\frac{V_{d s t}^{O C}(t) \cdot I_{d s t}(t) \cdot \eta_{\text {rate }}\left(I_{d s t}(t)\right)}{V_{s r c}^{O C}(t) \cdot I_{s r c}(t)},
$$

where $\eta_{\text {rate }}$ is the charging efficiency reflecting the rate-capacity effect, as will be stated in Section 3.1.2.

\section{PROBLEM DESCRIPTION}

\subsection{System Models}

\subsubsection{Switching converter}

We use a buck-boost converter as the voltage converter and charger to accommodate a wide range of $V_{s r C}^{C C}(t), V_{C T I}(t)$, and $V_{d s t}^{C C}(t)$. Efficiency of the buck-boost converter is largely determined by its input voltage, output voltage, and output current. We import an efficiency model of a PWM (pulse width modulation) buck-boost converter from [8]. The conversion efficiency $\eta$ is defined as:

$$
\eta=\frac{P_{\text {out }}}{P_{\text {in }}}=\frac{V_{\text {out }} \cdot I_{\text {out }}}{V_{\text {in }} \cdot I_{\text {in }}}=\frac{V_{\text {in }} \cdot I_{\text {in }}-P_{\text {converter }}}{V_{\text {in }} \cdot I_{\text {in }}},
$$

where $V_{\text {in }}$ and $V_{\text {out }}$ are input and output voltages; $I_{\text {in }}$ and $I_{\text {out }}$ are input and output currents; and $P_{\text {converter }}$ is the power consumption of the switching converter, respectively. The major sources of power consumption of the switching converter include conduction loss and switching loss in the switches, and controller power loss [8]. The power loss in the buck mode such that $V_{\text {in }}>V_{\text {out }}, P_{\text {converter }}$ is given

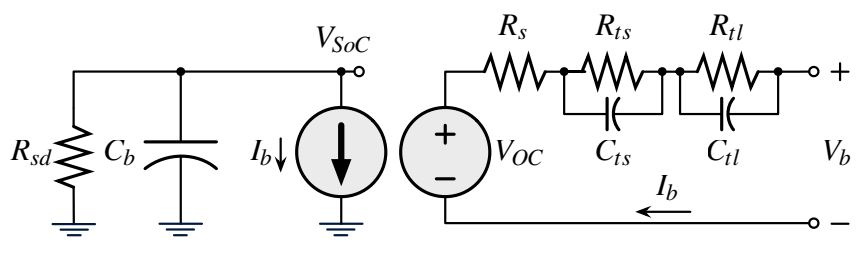

Figure 4: Li-ion battery equivalent circuit model.

by

$$
\begin{aligned}
P_{\text {converter }}= & I_{\text {out }}{ }^{2} \cdot\left(R_{L}+D \cdot R_{s w 1}+(1-D) \cdot R_{s w 2}+R_{s w 4}\right) \\
& +\frac{(\Delta I)^{2}}{12} \cdot\left(R_{L}+D \cdot R_{s w 1}+(1-D) \cdot R_{s w 2}+R_{s w 4}+R_{C}\right) \\
& +V_{\text {in }} \cdot f_{s} \cdot\left(Q_{s w 1}+Q_{s w 2}\right)+V_{\text {in }} \cdot I_{\text {controller }}
\end{aligned}
$$

where $D=\frac{V_{\text {out }}}{V_{\text {in }}}$ is the PWM duty ratio and $\Delta I=\frac{V_{\text {out }} \cdot(1-D)}{L_{f} \cdot f_{s}}$ is the maximum current ripple, $f_{s}$ is the switching frequency, $I_{\text {controller }}$ is the current flowing into the controller. The four switches in Figure 3 have series resistances $R_{s w 1 \cdots 4}$ and $Q_{s w 1 \cdots 4}$. The series resistances of the inductor and capacitor in Figure 3 are denoted by $R_{L}$ and $R_{C}$, respectively. The first and second terms of (4) are DC and AC conduction losses, respectively; the third term is the switching loss while the last term is the controller power consumption.

On the other hand, the power loss in the boost mode such that $V_{\text {in }}<V_{\text {out }}, P_{\text {converter }}$ is given by

$P_{\text {converter }}=$

$\left(\frac{I_{\text {out }}}{1-D}\right)^{2} \cdot\left(R_{L}+D \cdot R_{s w 3}+(1-D) \cdot R_{s w 4}+R_{s w 1}+D \cdot(1-D) \cdot R_{C}\right)$

$+\frac{(\Delta I)^{2}}{12} \cdot\left(R_{L}+D \cdot R_{s w 3}+(1-D) \cdot R_{s w 4}+R_{s w 1}+(1-D) \cdot R_{C}\right)$

$+V_{\text {out }} \cdot f_{s} \cdot\left(Q_{s w 3}+Q_{s w 4}\right)+V_{\text {in }} \cdot I_{\text {controller }}$,

where $D=1-\frac{V_{\text {in }}}{V_{\text {out }}}$ and $\Delta I=\frac{V_{\text {in }} \cdot D}{L_{f} \cdot f_{s}}$.

\subsubsection{EES banks}

In this paper, we focus on charge migration efficiency. We present a single source and a single destination migration assuming two representative EES elements: Li-ion batteries and supercapacitors, which can effectively explain fundamentals of charge migration without loss of generality. The above two EES elements have distinctive characteristics from each other. Typically, supercapacitors have advantages of relatively high power capacity and low internal resistance, but disadvantages of low energy capacity, high self-discharge rate, and a wide range of voltage variation depending on SoC, compared with batteries. Generally, supercapacitors are used as temporary energy storage for high intermittent power, while batteries are used as a long-term large-scale energy storage.

Battery models for the electronic systems have extensively been studied during the past few decades. Among those studies, the models based on electrochemical process modeling and analysis [9, 10], although accurate, are too complicated to be used for the systemlevel design of electronics. As we develop an mathematical formulation of the migration efficiency, a battery model in the form of an electric circuit is suitable for our purpose [11, 12].

We adopt the battery model introduced in [12] as shown in Figure 4. This includes a runtime-based model on the left as well as a circuit-based model on the right for accurate capture of the battery service life and I-V characteristics. We calculate the parameters of a 
Li-ion battery with the following non-linear equations:

$$
\begin{aligned}
v_{O C} & =b_{11} e^{b_{12} v_{S O C}}+b_{13} v_{S O C}^{3}+b_{14} v_{S O C}^{2}+b_{15} v_{S O C}+b_{16}, \\
R_{\text {series }} & =b_{21} e^{b_{22} v_{S O C}}+b_{23}, R_{t s}=b_{31} e^{b_{32} v_{S O C}+b_{33}}, \\
C_{t s} & =b_{41} e^{b_{42} v_{S O C}}+b_{43}, R_{t l}=b_{51} e^{b_{52} v_{S O C}}+b_{53}, \\
C_{t l} & =b_{61} e^{b_{62} v_{S O C}}+b_{63}, C_{b}=3600 \cdot \text { Capacity, }
\end{aligned}
$$

where $b_{i j}$ s are empirically extracted parameters from real pulsed discharging measurements, and Capacity denotes the nominal energy capacity of the battery.

The rate capacity effect of batteries specifies the fact that the available charge/discharge capacity (equivalently, the charge/discharge efficiency) decreases with the increase of charge/discharge current. In this paper, we will focus on the charging efficiency of the battery. Therefore, we use the Peukert's formula [13], an empirical equation to evaluate the relationship between the charging efficiency and the charging current, which is given by

$$
\eta_{\text {rate }}(I)=\frac{k}{I^{\alpha}}
$$

where $k$ and $\alpha$ are constants. Typically, the rate-capacity effect is not considered for supercapacitors, i.e., $\eta_{\text {rate }}=1$.

Similarly, we model the supercapacitor with circuit elements, too. The equivalent circuit model incorporates an equivalent series resistance which is small (about 50-100 $\mathrm{m} \Omega$ ) and a parallel leakage resistance which is typically in the order of $100 \mathrm{k} \Omega$.

\subsection{Formal Problem Statement}

In Figure 2, we control two independent variables, $V_{C T I}(t)$ and $I_{C T I}(t)$, at time $t$ for given OCVs $V_{s r c}^{O C}(t)$ and $V_{d s t}^{O C}(t)$, which can be derived through the SoC-OCV relation such as (6). We have dependent variables $I_{s r c}(t)$ and $I_{d s t}(t)$ that can be determined accordingly by (1), as well as the converter efficiency introduced in Section 3.1.1. We may control the two independent control variables by controlling $V_{C T I}(t)$ and $I_{d s t}(t)$ rather than $V_{C T I}(t)$ and $I_{C T I}(t)$, which is equivalent and more convenient because we can easily control $I_{d s t}(t)$ by setting the charger current.

The charge migration problem is defined as to transfer a given amount of charge, $Q$, from the source EES bank to the destination bank. We may define i) a time-unconstrained migration and ii) a time-constrained migration. The time-unconstrained migration is to transfer the charge in such a way that we have the minimum energy loss during the transfer with no time limit. A time-constrained migration is to transfer a given amount of charge within a given amount time, $T_{d}$. The charge migration problem can be formally described as an optimal control problem as follows, considering efficiencies of both the charger and discharger, rate capacity effect, as well as OCV variations of the storage banks.

Given Then initial source OCV $V_{s r c}^{O C}\left(T_{o}\right)$ and destination OCV $V_{d s t}^{O C}\left(T_{o}\right)$ at time $T_{0}$, the amount of charge to be migrated to the destination $Q$, and the relative deadline $T_{d}$. In the unconstrained charge migration problem, $T_{d}=\infty$.

Find The optimal CTI voltage $V_{C T I}(t)$ and charging current $I_{d s t}(t)$ $\left(T_{o}<t<T_{o}+T_{d}\right)$ during the charge migration process.

\section{Such that}

1) the amount of charge migrated to destination EES bank equals to $Q$, i.e., $\int_{T_{o}}^{T_{o}+T_{d}} I_{d s t}(t) \cdot \eta_{\text {rate }}\left(I_{d s t}(t)\right) d t=Q$.

2) the overall migration efficiency, given by

$$
\frac{\int_{T_{o}}^{T_{o}+T_{d}} V_{d s t}^{O C}(t) \cdot I_{d s t}(t) \cdot \eta_{\text {rate }}\left(I_{d s t}(t)\right) d t}{\int_{T_{o}}^{T_{o}+T_{d}} V_{s r C}^{O C}(t) \cdot I_{s r c}(t) d t},
$$

is maximized throughout the migration, or equivalently, the total amount of energy extracted from the source, given by $\int_{T_{o}}^{T_{o}+T_{d}} V_{s r c}^{O C}(t) \cdot I_{s r c}(t) d t$, is minimized.

\section{OPTIMIZATION METHOD}

In this section, we provide systematic solutions for the singlesource and single-destination charge migration. We consider both the time-unconstrained and time-constrained cases. We derive the optimal solution for the time-unconstrained case as stated in Section 4.1, and we derive a near-optimal solution for the time-constrained case considering the complexity of the solution method. As we loose the time constraint, the proposed near-optimal solution converges to the optimal solution as stated in Section 4.2.

\subsection{Time-Unconstrained Charge Migration}

First, we consider the instantaneous charge migration efficiency at time $t$. We are given OCVs of the source and destination, $V_{s r c}^{O C}(t)$ and $V_{d s t}^{O C}(t)$, respectively, and we have two control variables, $I_{d s t}(t)$ and $V_{C T I}(t)$. This problem is a specific case of the general problem stated in Section 3.2 as $T_{d} \rightarrow 0$. We maximize the instantaneous charge migration efficiency, given by:

$$
\begin{aligned}
& \max _{I_{d s t}(t), V_{C T I}(t)} \eta_{m}\left(I_{d s t}(t), V_{C T I}(t)\right) \\
= & \max _{I_{d s t}(t), V_{C T I}(t)} \frac{V_{d s t}^{O C}(t) \cdot I_{d s t}(t) \cdot \eta_{\text {rate }}\left(I_{d s t}(t)\right)}{V_{s r C}^{O C}(t) \cdot I_{s r c}(t)},
\end{aligned}
$$

We denote $I_{d s t}(t)$ and $V_{C T I}(t)$ that achieve the optimal charge migration efficiency as $I_{d s t, o p t}(t)$ and $V_{C T I, o p t}(t)$, respectively. They are functions of $V_{s r c}^{O C}(t)$ and $V_{d s t}^{O C}(t)$.

Generally speaking, maximization of the instantaneous migration efficiency is a quasi-convex (unimodal) optimization problem over $I_{d s t}(t)$ and $V_{C T I}(t)$ for reasonable $V_{s r c}^{O C}(t), V_{d s t}^{O C}(t)$ settings. For a higher $I_{d s t}(t)$, it is likely that the migration efficiency is low because of power dissipation on the battery internal resistance and the rate capacity effect. For a lower $I_{d s t}(t)$, it is likely that the migration efficiency is also low because of the low switching converter efficiency. We may exploit bisection-based algorithms using such quasi-convex property, which will make the solution quickly converge to the global optimal or a near global optimal solution. If the exact global optimal solution is required for all $\left(V_{s r c}^{O C}(t), V_{d s t}^{O C}(t)\right)$ pair settings, we may exploit branch and bound techniques here. We have an interesting observation that the optimal charging current $I_{d s t, o p t}(t)$ is larger when $V_{s r c}^{O C}(t)>V_{d s t}^{O C}(t)$, i.e., the overall system is in the buck mode. By contrast, $I_{d s t, o p t}(t)$ becomes smaller when $V_{s r c}^{O C}(t)<V_{d s t}^{O C}(t)$, i.e., the overall system is in the boost mode. This observation is useful in developing near-optimal algorithms in the time-constrained migration problem.

Now we consider the whole time-unconstrained charge migration process, as stated in Section 3.2, in which $V_{s r c}^{O C}(t)$ and $V_{d s t}^{O C}(t)$ keep changing with $t$. Typically, $V_{s r c}^{O C}(t)$ decreases while $V_{d s t}^{O C}(t)$ increases as time elapses since charge migrations from the source bank to the destination bank. The key idea to maximize overall migration efficiency, given by (8), is to maximize the instantaneous migration efficiency at every $t \in\left[T_{o}, T_{o}+T_{d}\right]$ according to the current $V_{s r c}^{O C}(t)$ and $V_{d s t}^{O C}(t)$, i.e., to perform charge migration with $I_{d s t, o p t}(t)$ and $V_{C T I, o p t}(t)$ derived from optimizing (9) at any time $t \in\left[T_{o}, T_{o}+T_{d}\right]$. The proposed algorithm guarantees the optimal overall migration efficiency if there is no leakage in the storage banks. In practice, we solve the charge migration problem in a discrete time space. We find the $I_{d s t, o p t}(t)$ and $V_{C T I, o p t}(t)$ according to the current $V_{s r c}^{O C}(t)$ and $V_{d s t}^{O C}(t)$ at each decision epoch (the beginning of each time slot), setting $I_{d s t}(t)=I_{d s t, o p t}(t)$ and $V_{C T I}(t)=V_{C T I, o p t}(t)$, and keep the same $I_{d s t}(t)$ and $V_{C T I}(t)$ within that time slot.

In order to implement the algorithm discussed above, online optimization is required at each decision epoch, which is a waste of time and energy. To overcome such a situation, we separate the charge migration algorithm into offline and online phases. We build a lookup table in offline, using a bisection method or a branch and 


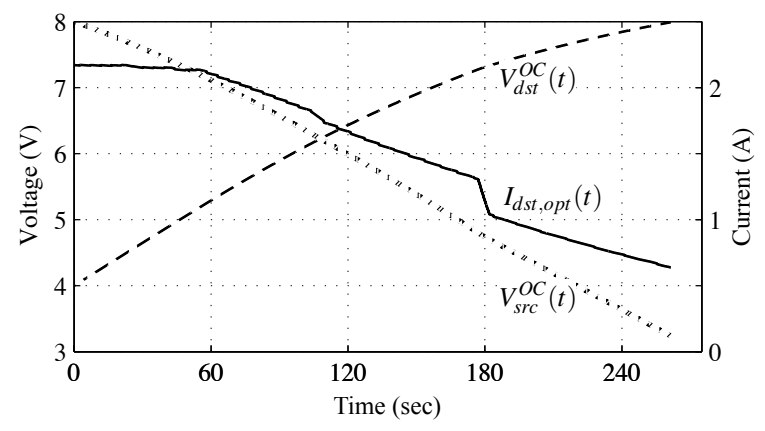

Figure 5: An example of optimal time-unconstrained charge migration.

bound method; while the online phase only needs to index the lookup table with current $V_{s r c}^{O C}(t)$ and $V_{d s t}^{O C}(t)$ at each decision epoch to perform optimal charge migration. The size of the lookup table can be kept small, since there are only two input variables, i.e., $V_{s r c}^{O C}(t)$ and $V_{d s t}^{O C}(t)$, and experimental results also back up that the granularity level of the lookup table does not affect much on the migration efficiency. Figure 5 illustrates an example of the optimal timeunconstrained charge migration, in which the source and destination are both supercapacitor banks, with the initial OCVs of $V_{s r c}^{O C}\left(T_{o}\right)=$ $8 \mathrm{~V}$ and $V_{d s t}^{O C}\left(T_{o}\right)=4 \mathrm{~V}$, respectively. The values of $V_{s r c}^{O C}(t), V_{d s t}^{O C}(t)$ and $I_{d s t}(t)\left(=I_{d s t, o p t}(t)\right)$ over time $t$ are shown in Figure 5.

\subsection{Time-Constrained Charge Migration}

There is a relative deadline $T_{d}<\infty$ in the time-constrained migration problem. There exists a minimal constant charging current to satisfy the timing constraint, which is denoted by $I_{d s t, \min }$, satisfying

$$
I_{d s t, \min } \cdot \eta_{\text {rate }}\left(I_{d s t, \text { min }}\right)=\frac{Q}{T_{d}} .
$$

This inspires us an uncomplicated yet effective heuristic for the timeconstrained charge migration problem as follows. At time $t$, if the optimal charging current $I_{d s t, o p t}(t)$ is greater than $I_{d s t, m i n}$, which is derived from indexing the lookup table built offline with the current $V_{s r c}^{O C}(t)$ and $V_{d s t}^{O C}(t)$, we set $I_{d s t}(t)=I_{d s t, o p t}(t)$. Otherwise, we set $I_{d s t}(t)=I_{d s t, \min }$. We continue to find the optimal $V_{C T I}(t)$, based on the current $V_{s r c}^{O C}(t), V_{d s t}^{O C}(t)$, and $I_{d s t}(t)$, using optimization methods.

Although this heuristic will guarantee that the charge migration "task" completes before the deadline, it still could be improved. Recall the observation stated in Section 4.1 about larger $I_{d s t, o p t}(t)$ when $V_{s r c}^{O C}(t)>V_{d s t}^{O C}(t)$ and smaller $I_{d s t, o p t}(t)$ when $V_{s r c}^{O C}(t)<V_{d s t}^{O C}(t)$. At the beginning of the charge migration, $V_{s r c}^{O C}(t)$ is higher, and $V_{d s t}^{O C}(t)$ is lower compared with that in the end the charge migration. Therefore, the optimal charging current at the beginning of the charge migration is usually higher compared with the optimal charging current at the end, as shown in Figure 5. We make the minimal charging current $I_{d s t, \text { min }}$ denoted by $I_{d s t, \text { min }}(t)$ also depend on time $t$, representing the minimal constant charging current required over time period $\left[t, T_{o}+T_{d}\right]$ to finish the remaining migration workload at time $t$. We update $I_{d s t, \min }(t)$ as charge migration proceeds such that

$$
I_{d s t, \min }(t) \cdot \eta_{\text {rate }}\left(I_{d s t, \min }(t)\right)=\frac{Q_{\text {rem }}(t)}{T_{o}+T_{d}-t},
$$

where $Q_{\text {rem }}(t)$ is the remaining workload at time $t$. At time $T_{o}<$ $t<T_{o}+T_{d}$, we set $I_{d s t}(t)=\max \left\{I_{d s t, \min }(t), I_{d s t, o p t}(t)\right\}$. In this way, we may be able to perform the charge migration with charging current $I_{d s t, o p t}(t)>I_{d s t, \min }(t)$ at the beginning of the charge migration, and gain some slack time. Therefore $I_{d s t, \min }(t)$ may decreases as $t$ increases because of the gained slack time, and $I_{d s t}(t)$ will keep decreasing as consequence. We can achieve the optimal (when the tim- ing constraint is relatively loose) or a near optimal overall migration efficiency using this improved heuristic. Practical implementation of the above heuristic could also be time slot based.

One critical issue in the implementation of the near-optimal heuristic may be caused by the fact that at time $t$, the charging current may not always equal to $I_{d s t, o p t}(t)$ for given $V_{s r C}^{O C}(t)$ and $V_{d s t}^{O C}(t)$ unlike the time-unconstrained problem because of the timing constraint. While the time-unconstrained problem has two input variables, the lookup table should have three input variables: $V_{s r c}^{O C}(t), V_{d s t}^{O C}(t)$ as well as $I_{d s t}(t)$ in the time-constrained problem. The output of the lookup table is the optimal $V_{C T I}(t)$. This results in that the size of the lookup table becomes huge. We propose the method of at any time $t$, using lookup table to only find $I_{d s t, o p t}(t)$, and then using high order curve fitting to find the (near) optimal $V_{C T I}(t)$ based on the current $V_{s r c}^{O C}(t)$, $V_{d s t}^{O C}(t)$ and $I_{d s t}(t)=\max \left\{I_{d s t, \min }(t), I_{d s t, o p t}(t)\right\}$. Fitting parameters of the high order curve fitting are determined offline while the online work for the controller is to find the fitted migration voltage value based on the fitting parameters and features. Details of the high order curve fitting are listed as follows:

- Separate curve fitting (different set of parameters) when the system is in the buck mode, i.e., $V_{s r c}^{O C}(t)>V_{d s t}^{O C}(t)$, and boost mode $V_{s r c}^{O C}(t)<V_{d s t}^{O C}(t)$.

- High order, i.e., there are nine features for curve fitting, $V_{s r c}^{O C}(t)$, $V_{d s t}^{O C}(t), I_{d s t}(t),\left(V_{s r c}^{O C}(t)\right)^{2},\left(V_{d s t}^{O C}(t)\right)^{2},\left(I_{d s t}(t)\right)^{2}, V_{s r c}^{O C}(t) \cdot V_{d s t}^{O C}(t)$, $V_{s r c}^{O C}(t) \cdot I_{d s t}(t)$ and $V_{d s t}^{O C}(t) \cdot I_{d s t}(t)$, and thus there will be ten fitting parameters including the constant term.

- In the training phase, optimal $V_{C T I}(t)$ value can be determined by optimization methods like branch and bound, and we use curve fitting results to determine the fitted value (near optimal) $V_{C T I, f i t}(t)$ online based on $V_{s r c}^{O C}(t), V_{d s t}^{O C}(t)$ and $I_{d s t}(t)$.

- We can set upper and lower bounds for the fitted value $V_{C T I, f i t}(t)$, which is effective in minimizing efficiency degradations.

The high order curve fitting adopted turns out to be effective in providing the near-optimal results with negligible online computation costs. When the system is in buck mode, the curve fitting results in only $0.02 \%$ efficiency degradation in average compared with the ideal case in which the optimal migration voltage for given $V_{s r c}^{O C}(t)$, $V_{d s t}^{O C}(t)$ and $I_{d s t}(t)$ is given in prior (which is not practical); and the degradation is $0.15 \%$ in boost mode.

\section{EXPERIMENTAL RESULTS}

In this section, we demonstrate the experimental results on single source single destination charge migration. Both time-unconstrained and time-constrained charge migration cases are analyzed, in Section 5.1 and 5.2, respectively. In each case, we demonstrate results for two representative charge migration scenarios: supercapacitor to supercapacitor, and supercapacitor to Li-ion battery. We compare the overall charge migration efficiency, from time $T_{o}$ to $T_{o}+T_{d}$ (with formula given by (8)), of the proposed solutions with baseline systems. The baseline systems use constant $I_{d s t}$ and $V_{C T I}$ in charge migration, independent of time $t$. We use Linear Technology LTM4607 converter as the charger and voltage converter model, and we obtain characteristics of Li-ion battery by measuring and extracting the parameters for the battery model given in Figure 4.

\subsection{Time-unconstrained Charge Migration}

The overall migration efficiency results of supercapacitor to supercapacitor, supercapacitor to Li-ion battery time-unconstrained charge migrations are given in Table 1 and Table 2, respectively. In these tables, the overall efficiencies of the optimal solution stated in Section 4.1 are shown in the first column, under the term "optimal". In the supercapacitor to supercapacitor charge migration case, the initial source and destination OCVs, $V_{s r c}^{O C}\left(T_{o}\right)$ and $V_{d s t}^{O C}\left(T_{o}\right)$, are $8 \mathrm{~V}$ and 
Table 1: Comparison of efficiencies in time-unconstrained supercapacitor to supercapacitor charge migration.

\begin{tabular}{|c||c||c|c|c|c|}
\hline \multirow{2}{*}{$T_{d}$} & \multirow{2}{*}{ Optimal } & \multicolumn{4}{|c|}{ Baseline } \\
\cline { 3 - 6 } & & & $V_{C T I}=1.0 \mathrm{~V}$ & $V_{C T I}=4.5 \mathrm{~V}$ & $V_{C T I}=8.0 \mathrm{~V}$ \\
\hline \multirow{4}{*}{$\infty$} & \multirow{3}{*}{$85.9 \%$} & $I_{d s t}=0.2 \mathrm{~A}$ & $44.5 \%$ & $57.8 \%$ & $56.4 \%$ \\
\cline { 3 - 6 } & $I_{d s t}=0.5 \mathrm{~A}$ & $60.4 \%$ & $76.2 \%$ & $69.5 \%$ \\
\cline { 3 - 6 } & & $I_{d s t}=1.0 \mathrm{~A}$ & $79.1 \%$ & $82.2 \%$ & $50.5 \%$ \\
\cline { 3 - 6 } & $I_{d s t}=2.0 \mathrm{~A}$ & $81.1 \%$ & $79.9 \%$ & $34.6 \%$ \\
\hline
\end{tabular}

Table 2: Comparison of efficiencies in time-unconstrained supercapacitor to $\mathrm{Li}$-ion battery charge migration.

\begin{tabular}{|c||c||c|c|c|c|}
\hline \multirow{2}{*}{$T_{d}$} & \multirow{2}{*}{ Optimal } & \multicolumn{4}{|c|}{ Baseline } \\
\cline { 3 - 6 } & & & $V_{C T I}=3.0 \mathrm{~V}$ & $V_{C T I}=4.0 \mathrm{~V}$ & $V_{C T I}=5.0 \mathrm{~V}$ \\
\hline \multirow{4}{*}{$\infty$} & \multirow{3}{*}{$82.1 \%$} & $I_{d s t}=0.2 \mathrm{~A}$ & $77.7 \%$ & $74.7 \%$ & $71.3 \%$ \\
\cline { 3 - 6 } & $I_{d s t}=0.5 \mathrm{~A}$ & $80.8 \%$ & $80.0 \%$ & $78.2 \%$ \\
\cline { 3 - 6 } & & $I_{d s t}=1.0 \mathrm{~A}$ & $69.5 \%$ & $70.4 \%$ & $69.7 \%$ \\
\cline { 3 - 6 } & & $I_{d s t}=2.0 \mathrm{~A}$ & $45.5 \%$ & $48.2 \%$ & $47.9 \%$ \\
\hline
\end{tabular}

$1 \mathrm{~V}$, respectively, and the target migration charge value $Q=720 \mathrm{C}$. The $V_{C T I}$ values in baseline systems are equal to $V_{s r C}^{O C}\left(T_{o}\right)=8 \mathrm{~V}$, $\left(V_{s r c}^{O C}\left(T_{o}\right)+V_{d s t}^{O C}\left(T_{o}\right)\right) / 2=4.5 \mathrm{~V}$ or $V_{d s t}^{O C}\left(T_{o}\right)=1 \mathrm{~V}$. In the supercapacitor to Li-ion battery case, we have $V_{s r c}^{O C}\left(T_{o}\right)=5 \mathrm{~V}, V_{d s t}^{O C}\left(T_{o}\right)=$ $3 \mathrm{~V}$, and $Q=800 \mathrm{C}$. The $V_{C T I}$ values in baseline systems are equal to $V_{s r c}^{O C}\left(T_{o}\right)=5 \mathrm{~V},\left(V_{s r c}^{O C}\left(T_{o}\right)+V_{d s t}^{O C}\left(T_{o}\right)\right) / 2=4 \mathrm{~V}$ or $V_{d s t}^{O C}\left(T_{o}\right)=3 \mathrm{~V}$. The charging current $I_{d s t}$ values used in baseline systems in both cases are $0.2 \mathrm{~A}, 0.5 \mathrm{~A}, 1 \mathrm{~A}$ or $2 \mathrm{~A}$.

As can be seen from Table 1 and Table 2, the optimal charge migration algorithm consistently outperforms baseline systems with constant $I_{d s t}$ and $V_{C T I}$. Most importantly, since there exists no systematic method determining the optimal constant $I_{d s t}, V_{C T I}$, it is not surprising for someone to design HEES systems with inappropriate $I_{d s t}, V_{C T I}$ values which yields very poor overall migration efficiency. The optimal solution shows up to $51.3 \%$ efficiency enhancement over a poorly configured baseline system, as Tables 1 and 2 show. More interesting result is that even the accidentally optimally configured baseline system is up to $3.7 \%$ less efficient than the optimal solution. This is because the optimal solution dynamically adjusts the $I_{d s t}, V_{C T I}$ values with time $t$ according to the current source and destination OCVs, to yield optimal overall migration efficiency.

\subsection{Time-Constrained Charge Migration}

The overall migration efficiency results of supercapacitor to supercapacitor, supercapacitor to Li-ion battery time-constrained charge migrations are given in Tables 3 and 4, respectively, with relative deadline $T_{d}$ values shown in the first column of each table. The overall efficiencies of the near-optimal solution stated in Section 4.2 are shown in the second column. The $V_{s r c}^{O C}\left(T_{o}\right), V_{d s t}^{O C}\left(T_{o}\right), Q$ and $V_{C T I}$ values of the baseline systems are exactly the same as those used in the time-unconstrained experiments in Section 5.1. On the other hand, we use charging current $I_{d s t}=I_{d s t \text {.min }}$ in the baseline systems corresponding to the given $T_{d}$ value, with $I_{d s t, \text { min }}$ defined in (10). In this way, charge migration will finish just before the deadline, with the smallest possible constant charging current.

As can be seen from Table 3 and Table 4, the proposed near optimal solution with lookup table and high order curve fitting consistently outperforms baselines systems under the same deadline constraint (the same $T_{d}$ value.) In the case when the deadline is very tight, the proposed solution, although forced to operate with charging current $I_{d s t}=I_{d s t, \min }$, outperforms baseline systems due to the use of curve fitting method to find the (near) optimal migration voltage. In the case when the deadline is loose, the proposed method has additional degree of freedom of choosing the optimal charging current. In this case, the overall migration efficiency of the proposed method will converge to global optimal derived in Section 5.1.
Table 3: Comparison of efficiencies in time-constrained supercapacitor to supercapacitor charge migration.

\begin{tabular}{|c||c||c|c|c|}
\hline \multirow{2}{*}{$T_{d}(\mathrm{~s})$} & \multirow{2}{*}{ Near-optimal } & \multicolumn{3}{|c|}{ Baseline } \\
\cline { 3 - 5 } & & $V_{C T I}=1.0 \mathrm{~V}$ & $V_{C T I}=4.5 \mathrm{~V}$ & $V_{C T I}=8.0 \mathrm{~V}$ \\
\hline 300 & $83.4 \%$ & $30.7 \%$ & $78.5 \%$ & $80.4 \%$ \\
\hline 500 & $85.9 \%$ & $42.8 \%$ & $82.3 \%$ & $81.0 \%$ \\
\hline 1,000 & $85.9 \%$ & $56.5 \%$ & $79.8 \%$ & $75.4 \%$ \\
\hline 2,000 & $85.9 \%$ & $61.4 \%$ & $70.8 \%$ & $61.6 \%$ \\
\hline
\end{tabular}

Table 4: Comparison of efficiencies in time-constrained supercapacitor to Li-ion battery charge migration.

\begin{tabular}{|c||c||c|c|c|}
\hline \multirow{2}{*}{$T_{d}(\mathrm{~s})$} & \multirow{2}{*}{ Near-optimal } & \multicolumn{3}{|c|}{ Baseline } \\
\cline { 3 - 5 } & & $V_{C T I}=3.0 \mathrm{~V}$ & $V_{C T I}=4.0 \mathrm{~V}$ & $V_{C T I}=5.0 \mathrm{~V}$ \\
\hline 1,000 & $73.2 \%$ & $72.2 \%$ & $72.7 \%$ & $72.0 \%$ \\
\hline 1,500 & $80.4 \%$ & $79.7 \%$ & $79.3 \%$ & $77.6 \%$ \\
\hline 3,000 & $82.1 \%$ & $80.8 \%$ & $78.9 \%$ & $75.7 \%$ \\
\hline 5,000 & $82.1 \%$ & $75.6 \%$ & $72.5 \%$ & $68.9 \%$ \\
\hline
\end{tabular}

\section{CONCLUSIONS}

Electrical energy storage (EES) systems have a great potential to enhance the power system efficiency. Hybrid EES (HEES) system is one of the most promising and practical ways to achieve highperformance and low-cost EES systems. This paper is the first paper that introduces the fundamentals of charge migration, which is one of three key operations in managing the HEES system, including problem definition, formulation and solution method targeting the best migration efficiency. This paper provided a systematic derivation of the optimal charge migration for the case of a single source and a single destination. We defined time-unconstrained and timeconstrained charge migration problems and derived the optimal and near optimal solutions, respectively. The experimental results show that the proposed solutions enhance the overall migration efficiency up to $51.3 \%$, compared with baseline charge migration methods.

\section{REFERENCES}

[1] H. T. Odum, "Energy quality and carrying capacity of the earth," Tropical Ecology, 1975.

[2] C. J. Cleveland, "Energy quality and energy surplus in the extraction of fossil fuels in the u.s.," Ecological Economics, 1992.

[3] H. Chen, T. N. Cong, W. Yang, C. Tan, Y. Li, and Y. Ding, "Progress in electrical energy storage system: A critical review," Progress in Natural Science, 2009.

[4] J. Baker and A. Collinson, "Electrical energy storage at the turn of the millennium," Power Engineering Journal, 1999.

[5] D. H. Doughty, P. C. Butler, A. A. Akhil, N. H. Clark, and J. D. Boyes, "Batteries for large-scale stationary electrical energy storage," The Electrochemical Society Interface, 2010.

[6] M. Pedram, N. Chang, Y. Kim, and Y. Wang, "Hybrid electrical energy storage systems," in ISLPED, 2010.

[7] F. Koushanfar, "Hierarchical hybrid power supply networks," in $D A C$, 2010.

[8] Y. Choi, N. Chang, and T. Kim, "DC-DC converter-aware power management for low-power embedded systems," IEEE T. on CAD, 2007.

[9] P. Rong and M. Pedram, "An analytical model for predicting the remaining battery capacity of lithium-ion batteries," IEEE T. on VLSI, 2006.

[10] D. Rakhmatov, "Battery voltage modeling for portable systems," $A C M$ TODAES, 2009.

[11] L. Benini, G. Castelli, A. Macii, E. Macii, M. Poncino, and R. Scarsi, "Discrete-time battery models for system-level low-power design," IEEE T. on VLSI, 2001.

[12] M. Chen and G. Rincon-Mora, "Accurate electrical battery model capable of predicting runtime and I-V performance," IEEE T. on Energy Conversion, 2006.

[13] D. Linden and T. B. Reddy, Handbook of Batteries. McGrew-Hill Professional, 2001. 